\title{
99 T CELL IMMUNOTHERAPIES TRIGGER NEUTROPHIL ACTIVATION TO ELIMINATE TUMOR ANTIGEN ESCAPE VARIANTS
}

'Daniel Hirschhorn*, 'Sadna Budhu, 'David Schröder, 'Lukas kraehenbuehl, 'AnneLaurent Flammar, ${ }^{1}$ Andrew Chow, ${ }^{1}$ Isabell Schulze, ${ }^{1}$ Sara Schad, 'Jacob Ricca, ${ }^{1}$ Billel Gasmi, ${ }^{1}$ Olivier De Henau, ${ }^{1}$ Levi Mangarin, ${ }^{2}$ David Redmond, ${ }^{1}$ Czrina Cortez, ${ }^{1}$ Cailian Liu, ${ }^{1}$ Aliya Holland, 'Mathieu Gigoux, 'Asrhi Arora, 'Katherine Panageas, ${ }^{3}$ Gabrielle Rizzuto, ${ }^{4}$ Jean Albrengues, ${ }^{4}$ Mikala Egeblad, 'Jedd Wolchok, 'Taha Merghoub. 'Memorial Sloan Kettering Cancer Center, New York, NY, USA; ${ }^{2}$ Cornell Medical School, New York, USA; ${ }^{3}$ University of California San Francisco, San Francisco, USA; ${ }^{4}$ Cold Spring Harbor Laboratories, Cold Spring Harbor, NY, USA

Background Targeted immune-based therapies such as adoptive $\mathrm{T}$ cell transfer (ACT) are often ineffective because tumors evolve over time and under selective pressure display antigen loss variant clones. A classic example in melanoma is de-differentiation and loss of expression of antigenic proteins. Therapies that activate multiple branches of the immune system may eliminate such escape variants

Methods Here we show that melanoma-specific CD4+ ACT therapy in combination with OX40 co-stimulation or CTLA-4 blockade can eradicate large melanoma tumors with clonal escape variants.

Results Early on-target recognition of melanoma antigens by adoptively transferred tumor-specific CD4+ $\mathrm{T}$ cells was required. Surprisingly, however, complete tumor eradication was partially dependent on neutrophils. Supporting these findings, extensive neutrophil activation and neutrophil extracellular traps were found in mouse tumors and in biopsies of melanoma patients treated with immune checkpoint blockade. Conclusions Our findings uncover a novel interplay between $\mathrm{T}$ cells mediating the initial tumor- and tissue-specific immune response, and neutrophils mediating tumor destruction of antigen loss variants.

Ethics Approval All tissues were collected at MSKCC following study protocol approval by the MSKCC Institutional Review Board. All mouse procedures were performed in accordance with institutional protocol guidelines at Memorial Sloan-Kettering Cancer Center (MSKCC) under an approved protocol.

http://dx.doi.org/10.1136/jitc-2021-SITC2021.099 